\title{
BUNCH COMPRESSOR II AT THE TESLA TEST FACILITY
}

\author{
M. Geitz, A. Kabel ${ }^{*}$, G. Schmidt, H. Weise \\ Deutsches Elektronen-Synchrotron \\ Notkestraße 85 \\ D-22607 Hamburg, Germany, \\ for the TESLA Collaboration
}

\begin{abstract}
In the TESLA Test Facility (TTF), short bunches with low emittance are needed for the operation of a free electron laser. Several stages of bunch compression by means of magnetic chicanes will be used to reach the required peak current. The second stage of the bunch compression system, bunch compressor II, has been taken into operation recently. We describe design and instrumentation of the bunch compressor II and communicate experiences made during commissioning.
\end{abstract}

\section{INTRODUCTION}

The TESLA Test Facility (TTF) aims to conduct a proofof-principle experiment for a self-amplified spontaneous emission free electron laser. (SASE FEL). For FEL operation, high peak currents and low transversal emittances are crucial. To reach these quality conditions, the bunch has to be compressed longitudinally after it leaves the gun. In the case of the TTF FEL experiment, this is done in magnetic chicanes. Bunch Compressor II is the only chicane to be used for the proof-of-principle experiment, while a further stage of FEL operation, using smaller wavelengths, higher energy and a longer undulator, requires another stage of compression (Bunch Compressor III) The low-energy stage Bunch Compressor I will not be used for FEL operation.

\section{BUNCH COMPRESSOR II}

BC II is located between accelerating modules \#1 and \#2 of the TTF, operating at a design energy of $140 \mathrm{MeV}$. It has been designed to compress a bunch of length $1 \mathrm{~mm}$ down to $250 \mu \mathrm{m} .[5,8]$

\subsection{Geometry}

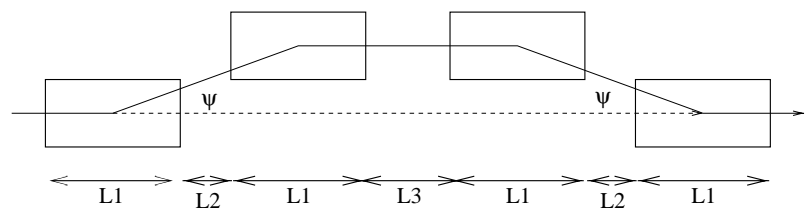

Figure 1: The geometry of a magnetic chicane

\footnotetext{
*E-Mail: andreas.kabel@ desy.de
}

Bunch Compressor II comprises four rectangular dipole magnets with field signature +--+ arranged with their entry faces in parallel and perpendicular to the beam entering the first magnet. This arrangement is easily seen to be overall dispersion-free to all orders. For BCII, the dimensions are $L_{1}=L_{2}=0.5 \mathrm{~m}, L_{3}=1.3 \mathrm{~m}$.

Optically, the chicane behaves like a drift space for the horizontal phase space plane (with an effective length exceeding the orbit length by $\Delta L=4 L_{1}\left(\frac{1}{\cos \psi}-\frac{\psi}{\sin \psi}\right)+$ $\left.\frac{2 L_{2} \sin ^{2} \psi}{\cos ^{3} \psi}\right)$ and as a strongly focusing element for the vertical phase space.

The vacuum chamber and the magnets have been designed to allow for an deflection angle of $\psi=17^{\circ} \ldots 21^{\circ}$. From geometry, the path length is $S(\psi)=\frac{4 L_{1} \psi}{\sin \psi}+\frac{2 L_{2}}{\cos \psi}+$ $L_{3}$. Thus, the linear matrix element $R_{56}=\frac{\partial S(\psi)}{\partial \delta}=$ $\tan \psi \frac{\partial S}{\partial \psi}=4 L_{1}\left(\frac{1}{\cos \psi}-\frac{\psi}{\sin \psi}\right)+2 L_{2} \frac{\tan ^{2} \psi}{\cos \psi}$ varies between $0.1594 \mathrm{~m}$ and $0.2546 \mathrm{~m}$.

From geometry, the offset in the symmetry point is

$$
h=2 L_{1} \frac{1-\cos \psi}{\sin \psi}+L_{2} \tan \psi,
$$

from which one has the maximum linear horizontal dispersion

$$
\eta_{x, \text { center }}=2 L_{1} \frac{\psi(1-\cos \psi)}{\tan \psi}+L_{2}\left(\tan \psi+\tan ^{3} \psi\right)
$$

In the case of BC II, $h=0.302 \mathrm{~m} \ldots 0.377 \mathrm{~m}$ and $\eta_{x, \text { center }}=0.310 \mathrm{~m} \ldots 0.393 \mathrm{~m}$.

The energy acceptance is $\delta_{a c c} \approx \frac{h_{\max }-h_{\min }}{\eta x, \text { center }}$, so one can expect transmission for beam energy variations of $\approx$ $\pm 10 \%$.

\subsection{Compression}

A bunch with a linear correlation element $d=\frac{\langle\delta x\rangle}{\sqrt{\left\langle x^{2}\right\rangle}}$ will thus be shortened by $-d R_{56}$. A linear correlation between $x$ and $\delta$ is achieved by shifting the acceleration module phase in front of the bunch compressor (normally accelerating from $E_{0}$ to $E_{0}+E_{1}$, where $E_{0} \approx 15 \mathrm{MeV}$ and $E_{1} \approx 85 \mathrm{MeV}$ for the commissioning run) by $\Delta \phi$. Then, $d=-\frac{E_{1} \sin \Delta \phi}{E_{0}+E_{1} \cos \Delta \phi}$ and the linear part of the bunch length vanishes for

$$
l_{\text {initial }}=d R_{56}
$$


. The quadratic term is $d_{2}=\frac{1}{1+\frac{E_{0}}{E_{1}} \cos \Delta \phi}$ and gives the minimum bunch length of $l_{\text {min }} \approx d_{2} R_{56} l_{\text {initial }} \frac{2 \pi^{2}}{\lambda^{2}}$, where $\lambda$ is the RF wavelength of $c / 1.3 \mathrm{Ghz}=0.231 \mathrm{~m}$.

Having the bunch travel through the chicane results in a phase slippage of $2 \pi \frac{S(\phi)-4 L_{1}-2 L_{2}-L_{3}}{\lambda}$ compared to going straight to the bunch compressor section. This is close to $\pi$ for the design value of $R_{56}$, which would result in a deceleration in the modules following the bunch compressor. A phase shifter-a length-adjustable waveguide-was installed to switch between straight and compression operation, allowing for on-crest acceleration from the 2 nd module on in both operation modes.

\subsection{Magnets}

The dipole magnets of the chicane require high fields and high field quality over a wide horizontal range. We use H-type magnets custom-manufactured by danfysik with a pole gap of $d=25 \mathrm{~mm}$. The pole width is $W=300 \mathrm{~mm}$, but the admissable width for the vacuum chamber is limited by spacers to $216 \mathrm{~mm}$. The offset of the horizontal positioning of the two inner dipoles was chosen as to fully exploit this range for variation of deflection angle. The maximum field strength is $0.375 \mathrm{~T}$, allowing for a maximum deflection angle of $23.7^{\circ}$ at $E=140 \mathrm{MeV}$.

The presence of quadrupole and higher moments in the field in the dispersive region will create additional transverse emittance. To avoid this, the field quality has to be quite high (specification values: sextupole: $<0.0043 \mathrm{~m}^{-2}$, decapole: $\left.<0.068 \mathrm{~m}^{-4}\right)$.

Electrically, the bending magnets are arranged as a series circuit, which guarantees equal field strengths, with individual trimcoils (giving up to $10 \%$ of main field value) in each magnet to correct leftover dispersion and horizontal steering errors.

The most crucial alignment issues are (1) equality of $L_{2}$ and its symmetric counterpart and (2) the tilt of the dipole magnets. Both errors would fail to close dispersion and would introduce angular kicks.

A total of four pairs ( $x$ and $y$ plane) steerer magnets with a possible correction of $\pm 3 \mathrm{mrad}$ have been inserted around the chicane and at the entrance and exit of the entrance and exit quadrupole triplet. They allow correction of all quad- and dipole-induced kicks within the tolerated misalignment.

\section{INSTRUMENTATION}

For commissioning bunch compressor II, it is important to get enough information to (1) steer the beam through the chicane, (2) reconstruct the bunch's phase space both longitudinally and transversally: the expected behavior for the longitudinal dynamics has to be checked, and it has to be ascertained that the emittance growth is within the expected limits.

The following instrumentation was installed in the bunch compressor II section or in connection with its installation:
- Toroids for transmission measurements

- An OTR foil in the dispersive region (middle of $L_{3}$ ), spanning the width of the vacuum chamber, thus allowing for energy spread measurement and steering the bunch through the chicane $[2,3]$

- Viewscreen and OTR foil at the exit of the chicane. They can be used for threading the bunch through the chicane.

- Synchrotron radiation outlet windows have been inserted into the vacuum chambers, allowing synchrotron radiation to be viewed for each bend in the dispersive section. The OTR foil at the exit of the chicane can be used as an outlet mirror, allowing to look into the most downstream bend.

- Stripline [4] and cavity [9, 10] beam position monitors (BPMs) at the entrance and exit of the chicane, respectively. In conjuction with the "cold" cavity BPM in the preceding module, one has two pairs of BPMs allowing to observe positional and angular offset at the entrance and exit of the chicane. Thus, one can check the closure of the orbit bump generated by the chicane.

- Interferometric bunch-length measurement: following the accelerating module \#2, OTR is generated in a foil and extracted using mirrors. A Martin-Puplett type interferometer is used to extract the autocorrelation function of the bunch's charge distribution. Pyroelectric detectors are used to measure the OTR's intensity.[1, 11]

- The chicane has a triplet of individually powered quadrupole magnets both upstream an downstream. In conjunction with OTR foils, they facilitate beam tomographic measurements, i.e. reconstruction of the transverse phase space.[6]

\section{COMMISSIONING}

We were able to steer a bunch of charge $10^{-9}$ As through the chicane. Excellent transmission could be reached; no current loss could be observed with the toroids. The transmission could be retained when varying the dipole current over a range of $\pm 6 \%$, which is in agreement with the energy acceptance given above. Save the synchrotron radiation observation, all instrumentation of the bunch compressor section could be commissioned.

\subsection{Compression}

Bunch length measurements upstream of the bunch compressor using a streak camera showed an initial bunch length of $3 \mathrm{~mm} . .4 \mathrm{~mm}^{1}$, which is off the design value of BC II.

\footnotetext{
${ }^{1}$ S. Schreiber, private communication
} 


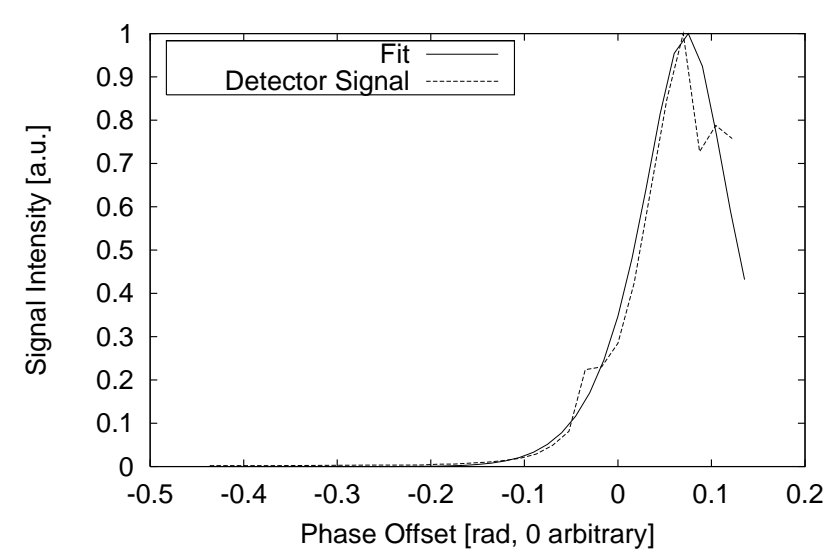

Figure 2: OTR Intensity measured with pyroelectric detectors and calculated intensity for purely gaussian bunches, see text

Thus, the minimum bunch length to be expected is around $700 \mu \mathrm{m}$ as opposed to the design value of $250 \mu \mathrm{m}$, which would be well above the minimum obtainable bunch length for $l_{\text {initial }}=1 \mathrm{~mm}$.

\subsection{Bunch Length Measurement}

Since the pyroelectric detectors cut off frequencies < $150 \mathrm{GHz}^{2}$ (corresponding to bunch length components of $>2000 \mu \mathrm{m}$ ), their signal can be used to find the off-crest phase giving the minimum bunch length. We observed a maximum in signal at a phase of $\Delta \phi \approx 12.1^{\circ}$ for an $R_{5} 6 \approx 0.20 \mathrm{~m}$, which gives, according to 3 an initial bunch length consistent with the streak camera measurements.

At this $\Delta \phi$ position, interferometric scans using the Martin Puplet interferometer was done. While the quality in terms of noise and resolution of the interferograms is good, the evaluation in terms of the bunch length still is inconclusive.

A more thorough evaluation of the amplitude measurements was done the following way: We assuming a coherent radiation intensity $I(\omega)$ as created by a Gaussian bunch with length $\sigma_{l}$ equal to its rms length (note that the compression process creates deviations from gaussian shape near maximum compression) of the bunch. Assuming a sharp cutoff-wavelength of the detector, one has $\int I(\omega) d \omega \propto \frac{1}{\sigma_{l}}\left(1-\operatorname{erf}\left(\sigma_{l} / \lambda_{\text {cutof } f}\right)\right)$. This intensity can be fitted (with parameters $\lambda_{c u t o f f}, R_{56}$, and $\sigma_{l, \text { initial }}$ ) to the actual readout of the pyroelectric detectors. $R_{56}$ is known to be $0.2 m \pm 10 \%$. One finds quite satisfactory agreement for $R_{56}=0.215 \mathrm{~m}, \sigma_{l, \text { initial }}=3.2 \mathrm{~mm}$ and $f_{\text {cutoff }}=150 \mathrm{GHz}$. This strongly indicates the presence of compression, but the evaluation of interferometric measurements would be necessary for further clarification.

\section{CONCLUSION}

The Bunch Compressor II at the TESLA Test Facility has been taken into operation; preliminary measurements strongly indicate compression of bunches. The results are consistent with the predicted behavior. The usability of the instrumentation has been demonstrated. However, due to the off-design values of initial bunch length emittance the design value for the final bunch length could not be reached in this run. Further measurements and further evaluation of measurement data is needed.

\section{REFERENCES}

[1] Ch. Berger, M. Geitz, B. Leissner, G. Schmidt, P. Schmueser, R. Siedling, M. Tonutti, "Bunch Length Measurements Using a Martin Puplett Interferometer”, This Conference, WEA131

[2] M. Castellano, V. A. Verzilov, Phys. Rev. ST Accel. Beams $1: 62801$ (1998)

[3] M. Castellano, V. A. Verzilov, LNF-98-017-P, June 1998.

[4] M. Castellano et al., "TESLA Test Facility Stripline Readout System", Conference Proceedings of the EPAC 96, Barcelona, June 1996

[5] D. A. Edwards (Ed.), TESLA Test Facility LINAC - Design Report, DESY Print March 1995, TESLA 95-01

[6] M. Geitz, G. Schmidt,"Phase Space Tomography at the TESLA Test Facility”, This Conference, WEA132

[7] M. Geitz, K. Hanke, P. Schmuser, Y. Y. Divin, U. Poppe, V. V. Pavlovskii, V. V. Shirotov, O.Y. Volkov, M. Tonutti, "A Hilbert Transform Spectrometer Using a High-T(C) Josephson Junction for Bunch Length Measurements at the TTF Linac”, DESY-TESLA-98-10, Apr 1998

[8] T. Limberg, A. Molodozhentsev, V. Petrov, H. Weise, "The Bunch Compression System at the TESLA Test Facility FEL.” DESY-TESLA-FEL-95-04A, 1995 and Nucl. Instrum. Methods A375 (1996) 322-324.

[9] R. Lorenz et al., "Measurement of the Beam Position in the TESLA Test Facility", Conference Proceedings of the LINAC96, Genevea, August 1996

[10] R. Lorenz et al., "First Operating Experiences of Beam Position Monitors in the TESLA Test Facility Linac ", Conference Proceedings of the PAC 97, Vancouver, May 1997

[11] J. Menzel, M. Tonutti, M. Geitz, P. Schmueser, Y. Y. Divin, U. Poppe, V. V. Pavlovskii, V. V. Shiritov, O. Y. Volkov "A Hilbert Transform Spectrometer Using a High $T_{C}$ Josephson Junction for Bunch Length Measurements at the TESLA Test Facility Linac", This Conference, WEA133

\footnotetext{
${ }^{2}$ Boris Leissner, private communication
} 